\title{
A Mathematical Modelling Approach to Design Microstrip Patch Antenna with Optimal Performance
}

\author{
R. J. Kavitha \\ Research Scholar \\ Visvesvaraya Technological University, Belagavi, \\ India
}

\author{
H. S. Aravind \\ Professor \& Head of the Department of Electronics \\ \& Communication Engineer, JSSATE, Bangalore, \\ India
}

\begin{abstract}
The design of microstrip patch antenna (MPA) demands reliable electro-magnetic interactions $(\mathrm{EmI})$ to meet the antenna design requirement. The antenna array faces problem with adjustment of design parameters such as elements dimensions, shape, spacing etc. Under this scenario, mathematical approach required to be essential to design the systematic design of array. But, the realistic models of arrays are computationally expensive in providing accuracy. Thus provide low cost computation, various approaches based on meta-heuristics like particle swarm optimization, genetic algorithms are proven effective against handling of such issues of pattern synthesis i.e., existence of side lobes. But, meta-heuristics requires thousands of function to evaluate the antenna design. In this paper, accurate mathematical modelling approach is presented to design Micro strip Patch Antenna with Optimal Performance. The mathematical model (using MATLAB) includes optimization of side lobes, optimization of radiation as well as reflection responses. The performance analysis of the approach is performed by considering the radiation, reflection coefficients, operating frequency and minimization of side lobes. The simulation outcomes of the antenna design give the reduced computational cost at $10 \mathrm{GHz}$ micro strip patch antenna.
\end{abstract}

\section{Keywords}

Computational cost, Micro strip patch antenna, Radiation, Reflection coefficients, Side lobes.

\section{INTRODUCTION}

The design of micro strip patch antenna (MPA) arrays need to be reliable for various electro-magnetic interactions (EmI) within the structures of antenna array to fulfill the requirements of the antenna design imposed over the array responses of radiations and reflection [1]. These electromagnetic interactions are composed of element coupling, impact of element environment, substrate finite size, feeding etc. Such impacts can only be reliably accounted for design process through typically discrete, full-wave, electro-magnetic simulations mainly by using complete antenna array module [2].

The array of an antenna involves different problems like adjustment of parameters like elements dimensions, array shape, array spacing, feeding location, phases etc. Under this case, the mathematical approach is found effective in designing the systematic antenna array [3]. But in real-time scenario, the computational cost of a simulation using mathematical approaches leads higher cost in designing of antenna array. The existing mathematical approaches consume higher cost because of involvement of more number of simulations in array model [4]. The approaches based on meta-heuristics like particle swarm optimization [5], genetic algorithms [6] are proven effective against handling of such issues of pattern synthesis i.e., existence of side lobes [7]. But, meta-heuristics requires thousands of function to evaluate the antenna design.

Thus, this paper aims to design an accurate computerized tool to design the micro strip patch antenna and introduces a mathematical approach for synthesis of patterns. The performance analysis of the approach is performed by considering the radiation, reflection coefficients, operating frequency and minimization of side lobes. The organization of the paper is done as: related work (section 2), system model of micro strip antenna (section 3), results analysis (section 4) and conclusion (section 5).

\section{RELATED WORK}

This section discusses the various researches in designing of antenna. Nowadays in wideband antennas, a lot of researches are going on, cause these are capable the satisfy the requirement of the high speed transmission for wireless communication terminals. A review work on micro strip antenna and its significant contribution towards communication system was performed by Kavita and Aravinda [8] which gives better idea of research gap in antenna technology of micro strip patch antenna. Towards same direction of research Tang et al. [9] have introduced a strip-helical antenna with a parasitic circular patch for circular polarization. By using this method the strip-helix are capable of working like an impedance bandwidth. Author Salih et al. [10] have explained the dual-band patch antenna for small form factor devices. The proposed antenna is fabricated on Rogers RO4350 board, the measured result have a good agreement with simulated ones. A dual-band circularly polarized antenna has been receiving much attention due to their distinct feature of single layer and single feeds. There are two different dissimilar radiators and two pairs of degenerate's mode TM01/TM10 and TM03/TM30 modes. In order to minimize the cost, mini authorized size, and easy integration author Zahang et al. [11] has proposed a dualfrequency band polarization method. This method achieves the radiation about 7.0and 5.9 in the two bands.

In the work of Katyal et al. [12], a transmission-line equivalent circuit technique is presented for analysis of multilayered micro strip patch antennas (MPA). The performance of the technique is validated by analysis of broadband antenna and found that the proposed method is capable for quick circuit level imitation and optimization. In the study of different type of printed antennas having different type of patch like, rectangular, square, triangular, circular, 
elliptical are suitable for $60 \mathrm{HZ}$ wireless application. For reduce overall size and to avoid lossy millimeter-wave connectors author Hannachi et al. [13-14] has proposed a key sight technologies, this given technology is very helpful of radio frequency design. In bandwidth improvement of an equilateral triangular micro strip patch antenna under differential excitation, the radiation directivity of TM11 mode is attached such a patch. For improved a lot of field distribution, Wang et al. [15] have presented an additional mod for both TM10and TM11 exited for radiation. Both virtual and measured result has been exhibit wide bandwidth and good presentation of radiation. Author Broker et al. [16] have introduced a linearly polarized dual-band patch antenna with low cross-polarization and autonomous band control. The procedure of antenna design is done on outlined and prototype for WiFi application. Finally the result are comes in the form of lower operating band and upper operating band could be shifted by up to $10 \%$ lastly.

In order to reduce the cross-polarization, low loss is applied to integrate the antenna array and further to improve the gain of the antenna array author Jing et al. [17] have presented a low temperature co-fired ceramic (LTCC) process. The given low temperature co-fired ceramic process has been used for fabricated and measured, the measured of 10- $\mathrm{dB}$ impedance and the gain of $18.62 \mathrm{~dB}$ at $61.5 \mathrm{GHz}$. The design of antenna and operation principle frequency and pattern-reconfigurable antenna whose based on a center-shorted micro strip patch antenna. Author Trong et al. [18] have introduced a centershorted micro strip mechanism by which DC bias voltage; both resonance frequencies can be changed simultaneously. The outcomes of [18] validate the proposed system with throughput and design procedure.

The works of $\mathrm{Li}$ et al. [19] have introduced a vertically integrated differential filtering antenna which consists of a differential-fed micro strip patch with U-shaped differential resonator. The proposed fabricated antenna behaves like frequency response for both gain and return loss. To generate millimeter-wave, Yao et al. [20] have explained a HermiteGussian (HG) method which is formed by using of four insetfed micro strip patch element also with a micro strip corporate feeding network. Through this work author has achieved accuracy in measurement and simulation. In Attaran et al. [21], a Rotman lens method is introduced where the length on the communication lines are not affected the progressive phase delay. Through this method complexity is minimized and performance parameters are maximized which finally gives low phase error of 0.450 in critical condition.

The work of Zhang et al. [22] introduced a micro strip patch antenna with the capabilities of bandwidth and harmonic suppression. Here, a pair of lemda/4 micros trio-line, the wide band property can be obtained by making useful use of the two resonances introduce by burning patch and non-radiating patch. The given prototype antenna is operating at $4.9 \mathrm{GHZ}$ is designed and fabricated, higher-order radiating modes has been effectively cancelled. In Sun et al. [23], a proximity coupled cavity backed patch antenna is described for long range RFID tag. The patch structure also offers a way to tune the resonant frequency of the antenna. The given antenna is achieved a gain of $5.7 \mathrm{dBi}$. In order to provide support towards two distinct operating frequencies author Smyth et al. [24] have given a novel dual-band micro strip patch antenna based on EBG integrated into its radiating edges. Through this dual antenna radiation tracked at $2.4 \mathrm{GHz}$ and $5.0 \mathrm{GHz}$ frequency.

Thus, in this paper the priority is given for designing an accurate computerized tool for micro strip patch antenna (MPA) and following introduced a mathematical approach that consists of optimized synthesis of patterns for MPA design.

\section{SYSTEM MODEL FOR MICROSTRIP PATCH ANTENNA}

In order to get the geometrical topology (architecture is shown in fig.1.) of the Micro strip patch antenna (MPA), the core component such as height of MPA (d1), width of MPA (d2), width of metal ground slot aperture (w1), length of metal ground slot aperture (u1), clot center to patch center (v1), length of open end stub, terminates the feed (v2), chamber length of the input micro strip (wc) and micro strip signal trace width (wo) are considered. The starting point (Sp) of the topology is initiated as [Spx, Spy] to arrive to the patch element with Xpatch [], Ypatch [] a schematic shown in fig. 1.

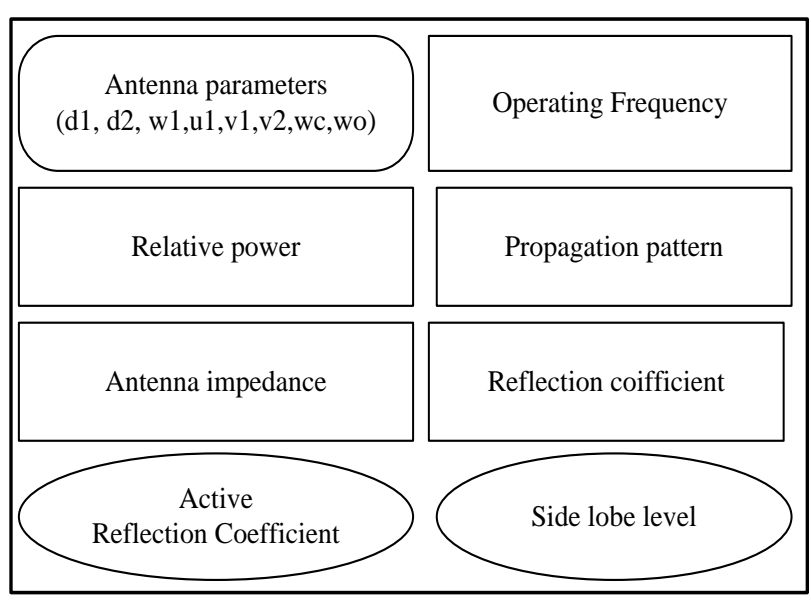

Figure 1 Architectural Model of Proposed Design

Here the information's of $\mathrm{d} 1, \mathrm{~d} 2 ; \mathrm{u} 1 ; \mathrm{v} 1, \mathrm{v} 2$; wc and wo are considered as input parameters. Later, the starting point (Sp) is defined corresponding to the coordinates of Xpath and Ypath. The Sp can be obtained by,

$$
\begin{aligned}
& \mathrm{Sp}=[\operatorname{Spx}, \operatorname{Spy}] \quad(\mathrm{Eq} 1) \\
& {[\text { Xpatch }]=\left[\operatorname{Spi}, \sum(\mathrm{Spi}+\mathrm{d} 2), \sum(\mathrm{Spi}+\mathrm{d} 2), \text { Spi, Spi }\right] \quad \text { at, }} \\
& \mathrm{i}=1 \quad(\mathrm{Eq} 2) \\
& {[\text { Ypatch }]=\left[\text { Spi,Spi } \sum(\mathrm{Spi}+\mathrm{d} 1), \sum(\mathrm{Spi}+\mathrm{d} 1),(\text { Spi1 })\right] \text { at, }} \\
& \mathrm{i}=2 \quad(\mathrm{Eq} 3)
\end{aligned}
$$

Further, the center localization of the patch $(\mathrm{Px}, \mathrm{Py})$ is computed by using eq. 4

$$
\begin{aligned}
& \mathrm{Px}=\sum\left(\left(\operatorname{Spi}+\left(\frac{\mathrm{d} \mathbf{2}}{2}\right)\right), \mathrm{i}=1\right. \\
& \mathrm{Py}=\sum\left(\left(\operatorname{Spi}+\left(\frac{\mathrm{d} \mathbf{1}}{2}\right)\right), \mathrm{i}=2 \quad(\mathrm{Eq} 4)\right.
\end{aligned}
$$




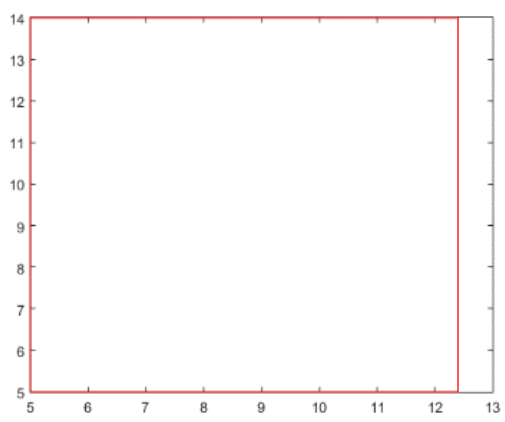

Figure 2 Patch with Xpatch[]. Ypatch[]

Based on these coordinates, a rectangle is plotted and for the same rectangle, patch center is determined.

$[\mathrm{Px} \mathrm{Py}] \leftarrow \mathrm{f}(\mathrm{Spi}, \mathrm{d} 1, \mathrm{~d} 2)$ at $\mathrm{i}=1,2 \quad(\mathrm{Eq} 5)$

Using this patch center, a center patch line is drawn.

i.e. $[p x-2 p x+v 1+v 2]$, [py py]

[px px], [2 py+d1/3+0.5] (Eq6)

Then the clot center $(\mathrm{Cc})$ is obtained, which is relative to the patch center.

$\mathrm{Cc}=([\mathrm{px}+\mathrm{v} 1 \mathrm{px}+\mathrm{v} 1],[\mathrm{py}-\mathrm{w} 0 / 2 \mathrm{py}+\mathrm{d} 1 / 3+0.5] \quad(\mathrm{Eq} 7)$

The distance for right angled triangle $\left(x_{t}\right)$ is obtained by using Eq8.

$$
\text { i.e. } x_{t}=\sqrt{w c^{2} / 2} \quad(\mathrm{Eq} 8)
$$

Then slot aperture of metal ground is calculated for both the Xpath and Ypath using Eq9.

xmetal_ground $=[\mathrm{px}+\mathrm{v} 1-\mathrm{w} 1 / 2 \quad \mathrm{px}+\mathrm{v} 1+\mathrm{w} 1 / 2 \quad \mathrm{px}+\mathrm{v} 1+\mathrm{w} 1 / 2$ $\mathrm{px}+\mathrm{v} 1-\mathrm{w} 1 / 2 \mathrm{px}+\mathrm{v} 1-\mathrm{w} 1 / 2]$

ymetal_ground $=[p y-u 1 / 2$ py-u1/2 py+u1/2 py+u1/2 py-u1/2]

Finally the labeling of the plot is done and outcome of the topology is shown in the fig. 3

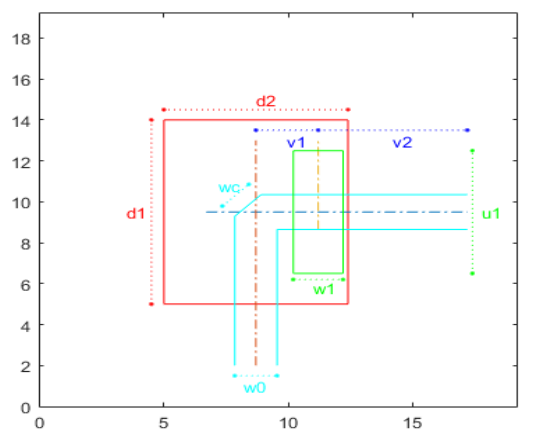

Figure 3 Default topology of MPA

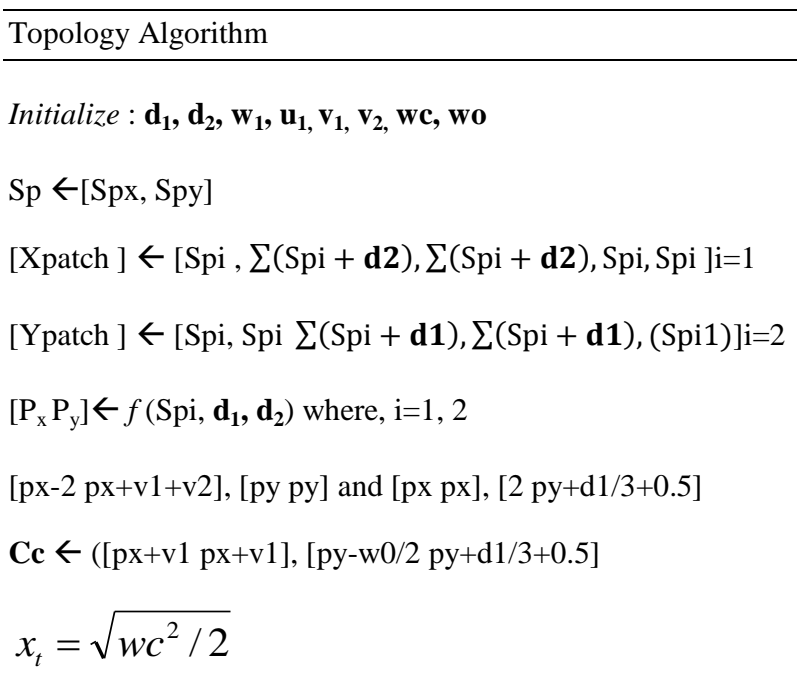

xmetal_ground $\leftarrow[\mathrm{px}+\mathrm{v} 1-\mathrm{w} 1 / 2 \mathrm{px}+\mathrm{v} 1+\mathrm{w} 1 / 2 \mathrm{px}+\mathrm{v} 1+\mathrm{w} 1 / 2$ $\mathrm{px}+\mathrm{v} 1-\mathrm{w} 1 / 2 \mathrm{px}+\mathrm{v} 1-\mathrm{w} 1 / 2]$

ymetal_ground $\leftarrow$ py-u1/2 py-u1/2 py+u1/2 py+u1/2 py$\mathrm{u} 1 / 2]$

\section{Synthesis of antenna array}

Algorithm for synthesis of antenna array

Initialize: $\mathrm{d} 1, \mathrm{~d} 2, \mathrm{w} 1, \mathrm{u} 1, \mathrm{v} 1, \mathrm{v} 2, \mathrm{wc}$, wo

$c \leftarrow 8 \times 10^{8}$

if $(\mathrm{i}=1 ; \mathrm{i}<=5 ; \mathrm{i}++)$ where $\mathrm{i}$ - is iteration

fc $\leftarrow$ nx $10^{9}$ where $\mathrm{n}=8,9,10,11,12$

$\lambda \leftarrow \mathrm{c} / \mathrm{fc}$

NR or NC $\leftarrow 2 / \operatorname{Sin} \theta_{b}$

$\mathrm{Op} \leftarrow$ NR_NC(c, fc [d1, d2, w1, u1, v1, v2, wc, wo $])$

$\mathrm{URA} \leftarrow \operatorname{Is}\left(\left[\begin{array}{lll}N R & N C\end{array}\right]\left[\begin{array}{ll}\lambda / 2 & \lambda / 2\end{array}\right]\right.$

$$
n U R A \leftarrow E w \times A w
$$

$\mathrm{NR} \leftarrow \mathrm{NR}+$

$\mathrm{NC} \leftarrow \mathrm{NC}-$

\section{Repeat for op}

In order to compute the relative power, the parameters like $\mathrm{d} 1$, d2, w1, u1, v1, v2, wc, wo are initialized. Later, to calculate the carrier frequency (fc), the signal propagation speed (c) is considered. Then, the wavelength $(\lambda)$ is obtained by dividing signal propagation speed (c) with carrier frequency (fc). 
i.e., $\lambda=\mathrm{c} / \mathrm{fc}$

(Eq10)

Further, the size of array along with elevation and azimuth direction can be formulated by required beam width. For the half wavelength spacing, the number of elements along with certain direction can be given as;

$\mathrm{NR}$ or $\mathrm{NC}=2 / \operatorname{Sin} \theta_{b}$

In Eq.11, the value of $\theta_{b}$ represents the beam width along that direction

The other parameters (Op) like azimuth cutoff and elevation cut off can obtained by following equation.

$$
\mathrm{Op}=\mathrm{NR} \_\mathrm{NC}(\mathrm{c}, \mathrm{fc}[\mathrm{d} 1, \mathrm{~d} 2, \mathrm{w} 1, \mathrm{u} 1, \mathrm{v} 1, \mathrm{v} 2, \mathrm{wc}, \mathrm{wo}])(\mathrm{Eq} 12)
$$

Then, the uniform rectangular array (URA) is considered as the integration of two separable uniform Line arrays (ULA) and designed the windows for both the elevation and azimuth direction through digital filer design methods. Then the URA developed by identical sensor elements can be given as:

i.e., URA $=$ Is $\left(\left[\begin{array}{lll}N R & N C\end{array}\right]\left[\begin{array}{ll}\lambda / 2 & \lambda / 2\end{array}\right]\right.$

In above Eq13, Is - indicates the identical sensor element

On assigning the weights to the array following Eq.14 is obtained.

i.e., $n U R A=E w \times A w \quad(\mathrm{Eq} 14)$

Where nURA indicates the new URA, Ew indicates the elevation weight and Aw represents the Azimuth weight. Later the comparison among the new URA and previous URA.

In antenna technology the side lobes are the local maxima or lobes of the far field radiation pattern which are not the main lobes. Here, the side lobe level of the new URA is compared with the previous design. However, the new URA does not meet the requirements and hence trial and error method is applied to NR and NC parameters.

i.e., $\mathrm{NR}=\mathrm{NR}+$

$\mathrm{NC}=\mathrm{NC}-$

Then obtained values of NR and NC are updated to get the optimized design results.

\section{RESULT ANALYSIS}

The design of Micro strip patch antenna is performed by using MATLAB software and its obtained results are compared. The Fig.4 indicates the resulting beam pattern for look directions ranging from less than $-30^{\circ}$ to $0^{0}$ azimuth and elevation degrees and keeps null at $-40^{\circ}$.
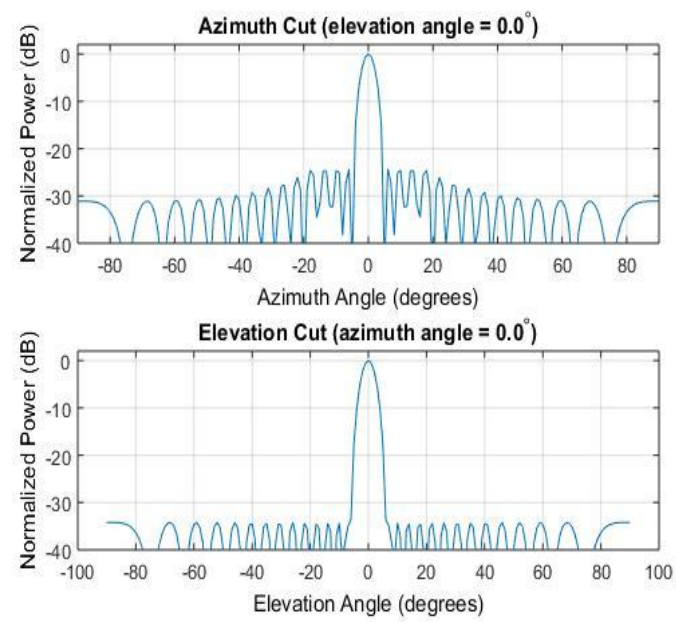

Figure 4 Beam patterns of azimuth angle and elevation angle

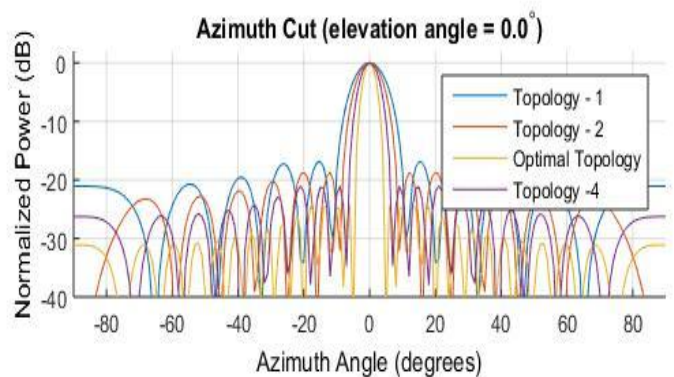

Topology -4

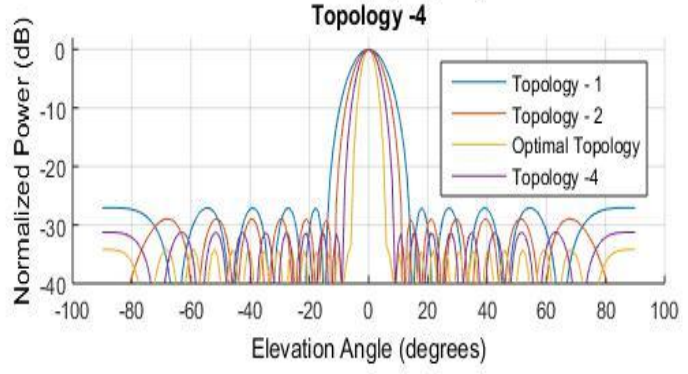

Figure 5 Synthesis of beam patterns with different methods

The fig. 5, gives the synthesis of array corresponding to topology1, topology2, topology4 and optimal topology with respect to bandwidth. In this, the array of topology1 is exceeding the required bandwidth of desired pattern than the topology 2, 4 and optimal topology. But the side lobes are higher than that of desired pattern. These side lobes can be reduced by using a windowing operation to array. As URA is considered to be the combination of two different uniform linear arrays (ULA), the window can be designed independently along with both elevation and azimuth directions by using filter designing models. In the fig.4, resulting side lobe level is compared with four different topologies. It is found that side lobe level of optimal topology is lower than that of topology 1, topology 2 and topology 4 . 


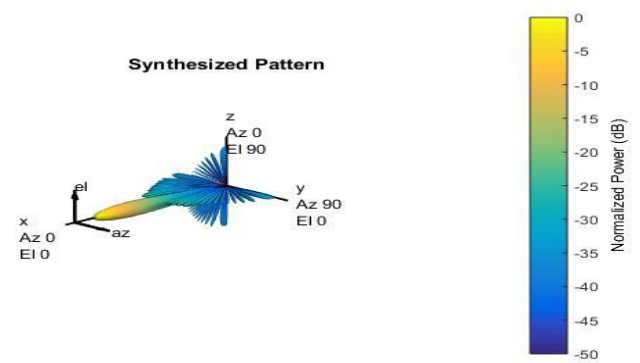

Figure 6 Synthesis of Design Pattern (3D)

The synthesis of design pattern is represented in fig.6. The 3D patterns of radiations consists of some symmetries for both azimuth and elevation cuts. Thus, the patterns obtained through uniform rectangular array (URA). From the fig.5, found that there is no energy is radiated towards back of array, where bandwidth and side lobe level of synthesized pattern match the desired specification. This indicates the synthesized 3D pattern.

The antenna input impedance is a frequency dependent quality. The plot represented in below fig.7, indicates the micro strip antenna over frequency band. The reactance and resistance trace varies with respect to frequency. The variation can be seen that the reactance values are negative before the resonance and the same value is positive after the resonance. This kind of reactance is known as series resonance. If the impedance curve reaches from positive to negative reactance and is known as parallel resonance. Both the resistance and reactance are fully different as resistance does not depends on frequency while reactance does. The resistance does not cause phase shift while reactance causes phase shift of $90^{\circ}$ among voltage and current. In the below Fig.6, resistance almost stays at positive value while reactance stays at negative value during resonance and reaches positive after resonance.

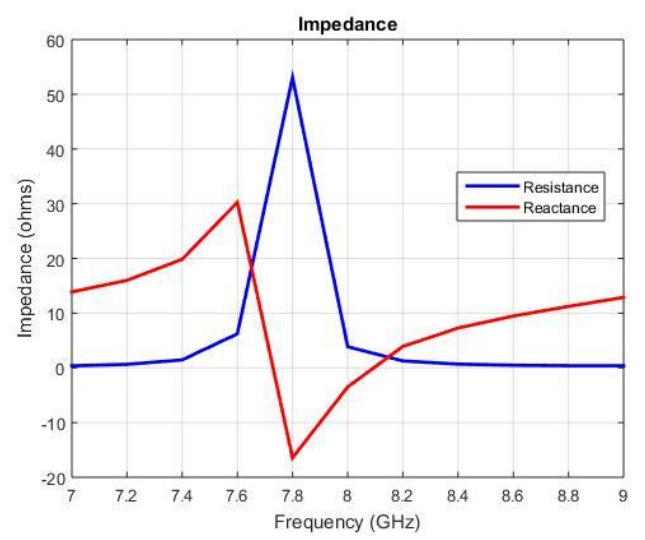

Figure 7 Analysis of Antenna over Frequency Band

The reflection coefficient (in Fig.8) of antenna indicates the relative fraction of the incident Radio frequency (RF) power which is reflected back because of impedance mismatch. The impedance mismatch is the difference among the antenna input impedance and the transmission line characteristic impedance (reference impedance). The reflection coefficient is also indicated the antennas operating bandwidth. The antenna bandwidth is the frequency band over which the reflection coefficient magnitude is $<-10 \mathrm{~dB}$.

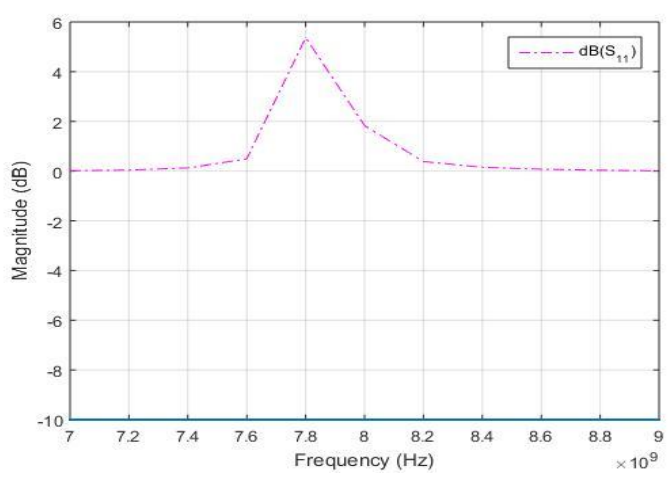

Figure 8 Reflection coefficients with respect to magnitude

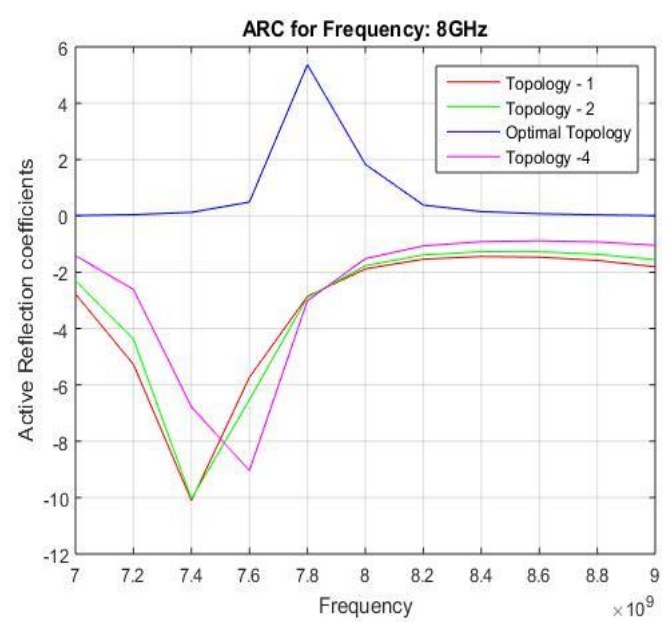

Figure 9 Active reflection coefficients with respect to different topologies

In Fig.9, active reflections coefficients of four different topology are compared corresponding to frequency are shown, where optimal topology acquired positive value than other topology1, topology 2 and topology4.

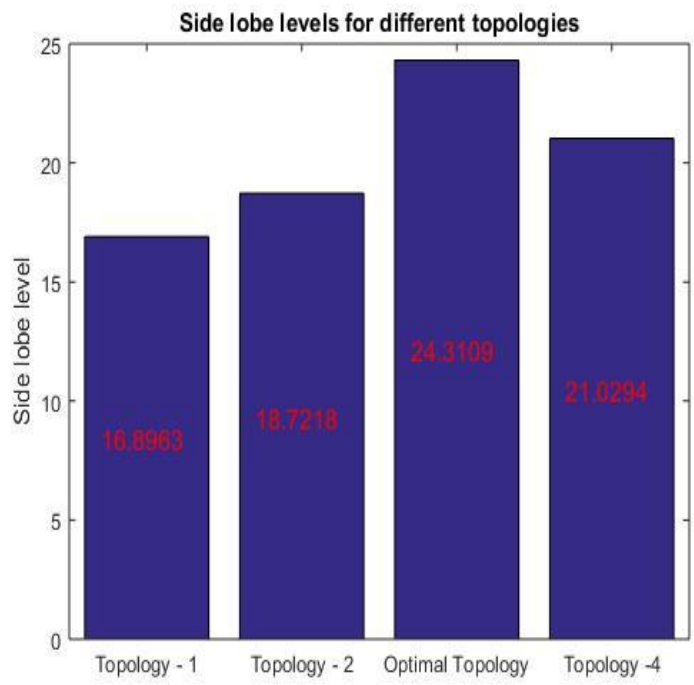

Figure 10 Side lobe levels for different methods

The side lobe level of an array indicates the far field radiation pattern and these are not the main lobes. The value of side lobe increases with decreases in the bandwidth. For a good array pattern, the value of side lobe level must be higher. In 
the above Fig.10, the optimal topology exhibit 24.3109 which is quite higher than topology1 (16.8963), topology2 (18.7218), and topology4 (21.0294).

\section{CONCLUSION}

This paper has introduced a mathematical approach to simulate the design of the linear micro strip path antenna (MPA). From the analysis of the approach it is been concluded that the approach allows significantly to control both the radiation as well as reflection coefficient through the element geometry design and identical sensor element. The implementation of this mathematical approach allows minimizing the cost of antenna to low level corresponding to some EMI of array antenna.

\section{REFERENCES}

[1] C.A. Balanis, Antenna theory, 3rd ed., Wiley Inter science, 2005.

[2] R.J. Mailloux, Phased array antenna handbook, 2nd ed., Artech House, 2005

[3] S. Koziel and S. Ogurtsov, "Antenna design using variable fidelity electromagnetic simulations," Int. J. Applied Electromagnetics and Mechanics, vol. 43, pp. 169-183, 2013.

[4] Koziel, Slawomir, et al. "Variable-fidelity electromagnetic simulations and co-kriging for accurate modeling of antennas." IEEE Transactions on Antennas and Propagation 61.3 (2013): 1301-1308.

[5] M.M. Khodier and C.G. Christodoulou, "Linear array geometry synthesis with minimum sidelobe level and null control using particle swarm optimization," IEEE Trans. Antennas Prop., vol. 53, no. 8, 2674-2679, Aug. 2005.

[6] F.J. Ares-Pena, A. Rodriguez-Gonzales, E. VillanuevaLopez, and S.R. Rengarajan, "Genetic algorithms in the design and optimization of antenna array patterns," IEEE Trans. Antennas Prop., vol. 47, pp. 506-510, Mar. 1999.

[7] D.W. Boeringer, D.H. Werner, and D.W. Machuga, "A simultaneous parameter adaptation scheme for genetic algorithms with application to phased array synthesis," IEEE Trans. Antennas Prop., vol. 53, pp. 356-371, Jan. 2005.

[8] Kavitha, R.J. and Aravinda, H.S., 2017. Reviewing the Effectiveness of Contribution of Microstrip Antenna in the Communication System. Open Journal of Antennas and Propagation, 5(02), p.47.

[9] Tang, Xihui, Yejun He, and Botao Feng. "Design of a Wideband Circularly Polarized Strip-Helical Antenna With a Parasitic Patch." IEEE Access 4 (2016): 77287735 .

[10] Salih, Ahmad A., and Mohammad S. Sharawi. "A DualBand Highly Miniaturized Patch Antenna." IEEE Antennas and Wireless Propagation Letters 15 (2016): 1783-1786.

[11] Zhang, Jin-Dong, et al. "CP patch antenna with controllable polarisation over dual-frequency bands." IET Microwaves, Antennas \& Propagation (2016).

[12] Katyal, Ankita, and Ananjan Basu. "Analysis and optimisation of broadband stacked MsAs using transmission line model." IET Microwaves, Antennas \& Propagation (2016).

[13] Hannachi, Chaouki, and Serioja Ovidiu Tatu. "Performance comparison of $60 \mathrm{GHz}$ printed patch antennas with different geometrical shapes using miniature hybrid microwave integrated circuits technology." IET Microwaves, Antennas \& Propagation (2016).

[14] Wang, Jianpeng, Qianwen Liu, and Lei Zhu. "Bandwidth Enhancement of a Differential-Fed Equilateral Triangular Patch Antenna via Loading of Shorting Posts." IEEE Transactions on Antennas and Propagation 65.1 (2017): 36-43.

[15] Brocker, Donovan E., et al. "Miniaturized Dual-band Folded Patch Antenna with Independent Band Control Utilizing an Interdigitated Slot Loading." IEEE Transactions on Antennas and Propagation (2016).

[16] Jin, Huayan, et al. "High-gain low-cross-polarization 60$\mathrm{GHz}$ LTCC patch antenna array with differential-fed and soft-surface structures." Microwave Conference (APMC), 2015 Asia-Pacific. Vol. 1. IEEE, 2015.

[17] Zhang, Xiu Yin, et al. "Low-Profile Dual-Band Filtering Patch Antenna and Its Application to LTE MIMO System." IEEE Transactions on Antennas and Propagation 65.1 (2017): 103-113.

[18] Nguyen-Trong, Nghia, Leonard Hall, and Christophe Fumeaux. "A frequency-and pattern-reconfigurable center-shorted MsA." IEEE Antennas and Wireless Propagation Letters 15 (2016): 1955-1958.

[19] Li, Lin, and Gang Liu. "A Differential MsA With Filtering Response." IEEE Antennas and Wireless Propagation Letters 15 (2016): 1983-1986.

[20] Yao, Haohan, et al. "Patch Antenna Array for the Generation of Millimeter-Wave Hermite-Gaussian Beams." IEEE Antennas and Wireless Propagation Letters 15 (2016): 1947-1950.

[21] Attaran, Ali, Rashid Rashidzadeh, and Ammar Kouki. "60 GHz Low phase error rotman lens combined with wideband MsA array using LTCC technology." IEEE Transactions on Antennas and Propagation 64.12 (2016): 5172-5180.

[22] Zhang, Jin-Dong, et al. "A Compact Microstrip-Fed Patch Antenna With Enhanced Bandwidth and Harmonic Suppression." IEEE Transactions on Antennas and Propagation 64.12 (2016): 5030-5037.

[23] Sun, Hu, Bo Tao, and Omar M. Ramahi. "Proximity Coupled Cavity Backed Patch Antenna for Long Range UHF RFID Tag." IEEE Transactions on Antennas and Propagation 64.12 (2016): 5446-5449.

[24] Smyth, Braden P., Stuart Barth, and Ashwin K. Iyer. "Dual-Band Microstrip Patch Antenna Using Integrated Uniplanar Metamaterial-Based EBGs." IEEE Transactions on Antennas and Propagation 64.12 (2016): 5046-5053. 